\title{
Within - and between - Session Reliability of the Spider Drill Test to Assess Change of Direction Speed in Youth Tennis Athletes
}

\author{
Jessica Huggins ${ }^{1}$, Paul Jarvis ${ }^{1}$, Jon Brazier ${ }^{2}$, Yianni Kyriacou ${ }^{1}$ and Chris Bishop ${ }^{1 *}$ \\ ${ }^{1}$ London Sport Institute, Middlesex University, UK \\ ${ }^{2}$ Department of Sport and Exercise Science, City and Islington College, UK
}

*Corresponding author: Chris Bishop, London Sport Institute, Middlesex University, Allianz Park, Greenlands Lane, NW4 1RL, UK, E-mail: C.Bishop@mdx.ac.uk

\begin{abstract}
Agility or Change of Direction Speed (CODS) is a critical physical attribute in a sport such as tennis, which is categorised by frequent and multiple changes of direction. Recently, a CODS test called the 'spider drill' has been used to assess tennis athletes' ability to change direction. To the authors' knowledge, no study has solely assessed its reliability and compared this with other commonly-used CODS tests; thus, this was the aim of the study. Ten nationally ranked youth tennis athletes (age: $15.1 \pm 2.6$; mass: 66.4 $+17.2 \mathrm{~kg}$; height: $163.0+16.2 \mathrm{~cm}$ ) completed three trials of the spider drill, modified t-test and pro-agility test on two separate testing occasions. All CODS tests had low typical percentage error, both within-sessions $(\mathrm{CV}=1.8-4.1 \%)$, and between session ( $\mathrm{CV}=1.2-3.7 \%)$. The SEM was also consistent within tests both within- and between- testing sessions. Within-session test-retest consistency illustrates strong reliability for the spider drill (ICC $=0.93,0.95)$, modified t-test (ICC $=0.79,0.83)$, however for pro-agility session 2 fell outside of the accepted threshold (ICC $=0.88,0.69$ ). These trends were similar when assessing between-session consistency, with both the spider drill and modified t-test providing high levels of reliability (ICC $=0.95$ and 0.97 respectively). However, the pro-agility fell outside of the accepted threshold (ICC $=0.66$ ), with $95 \%$ confidence intervals wide-ranging in nature $(95 \% \mathrm{Cl}: 0.11-0.9)$. These results suggest that the spider drill and modified t-test are both reliable tests when measuring CODS within youth tennis athletes. Strength and conditioning practitioners could consider changes in excess of $\pm 1.1 \%$ as meaningful (based off the SDD) when assessing CODS through the spider drill or modified t-test within youth tennis athletes.
\end{abstract}

\section{Keywords}

CODS, Change of direction, Reliability, Spider drill, Modified t-test, Pro-agility

\section{Introduction}

Agility has been identified as key to athletic success within numerous intermittent sports [1-5], with tennis being a prime example [6-8]. Within typical tennis match play, literature reports the average point length to last less than 10 seconds, with an average of 4 changes of direction per point $[9,10]$. Proficient movement in multiple directions, especially the lateral plane of motion is required [7,11], and this is supported by time motion analysis data which has shown elite players average four changes in direction per point and approximately 1000 changes in direction per match [7]. Additionally, Roetert, et al. [8] also concluded that agility was the best physical indicator to selection ranking amongst elite players. Considering agility is an integral component to athletic success in tennis, Strength and Conditioning ( $\mathrm{S} \& \mathrm{C}$ ) coaches need to be able to successfully assess a player's performance for this physical attribute. The importance of a field-based assessment throughout the year has proven beneficial to an athlete's development, as coaches can then accurately monitors an athletes progress and highlight strengths and weaknesses in order to tailor training programmes accordingly [10-12].

Despite its importance towards the tennis population, the definition of agility is often the subject of debate amongst the sport science community [13-16]. Within the literature, agility has been referred to by several definitions $[13,14,16-20]$. The most recent definition of agility describes elements of decision making

Citation: Huggins J, Jarvis P, Brazier J, Kyriacou Y, Bishop C (2017) Within - and between - Session Reliability of the Spider Drill Test to Assess Change of Direction Speed in Youth Tennis Athletes. Int J Sports Exerc Med 3:074. doi.org/10.23937/2469-5718/1510074

Received: May 25, 2017; Accepted: September 18, 2017; Published: September 20, 2017

Copyright: (c) 2017 Huggins J, et al. This is an open-access article distributed under the terms of the Creative Commons Attribution License, which permits unrestricted use, distribution, and reproduction in any medium, provided the original author and source are credited. 
and perceptual motor skills and has therefore caused many to suggest that some field-based assessments used by $S \& C$ coaches are inappropriate due to their lack of response to a stimulus $[13,14,21]$. However, it should be noted that recreating the 'decision-making' aspect of agility may in fact be incognisant. A review by Kovacs [7] highlights how every point in tennis is vastly different in nature, rendering the cognitive and perceptual demands as inconsistent. As a result of this, Change of Direction Speed (CODS) tests are considered favourable [15].

Two commonly used CODS assessments are the modified t-test and pro-agility $[11,22,23]$. Both are argued to be suitable and practical methods for the assessment of CODS performance of tennis players due to their emphasis on both linear and lateral movement patterning, acceleration and subsequent deceleration, of which is considered highly important for tennis performance $[6,7,11]$. Equally, both assessments cover a total distance of $20 \mathrm{~m}$, which may be pertinent to tennis as distances covered tend not to exceed $12 \mathrm{~m}$ [23]. However, though these two CODS assessments are heavily documented within the literature to produce reliable and sufficient results $[15,22,23]$, to the authors' knowledge, they have not been examined specifically amongst the tennis population. Additionally, a less wellknown CODS assessment is gaining popularity among the United States Tennis Association (USTA); the spider drill $[8,12,24,25]$. Though Eriksson, et al. [12] acknowledges that the movement patterns observed within this drill are very much like the movement patterns seen within tennis match play, at present there appears to be limited recognition to the application of the spider drill within the literature, particularly in respect to its reliability within youth tennis athletes $[8,24,25]$.

As CODS is considered key to athletic success among tennis players $[6-8,11]$, it is within the interest of S\&C coaches to select the most appropriate field-based assessment that can mimic the movement patterns of the sport and monitor the effects of training amongst athletes year-round $[11,12,21]$. Therefore, the primary aim of this study is to conduct a direct evaluation of all three previously mentioned CODS assessments (spider drill, modified t-test and pro-agility) in respect to their test retest reliability within elite youth tennis athletes, and to identify the Smallest Detectable Difference (SDD) between testing sessions.

\section{Methods}

\section{Approach to the problem}

In order to fulfil the requirements of this study, nationally ranked tennis athletes were required to attend three separate testing sessions. Session 1 was a familiarisation session, whereby participants were subjected to the experimental conditions. This enabled all participants to practice each of the CODS assessments 5 times, with a full verbal explanation and visual demonstration provided. Sessions 2 and 3 were data collection sessions, where by all subjects completed three trials for each of the CODS tests.

\section{Subjects}

Ten elite youth tennis athletes (age: $15.1 \pm 2.6$; mass: $66.4+17.2 \mathrm{~kg}$; height: $163.0+16.2 \mathrm{~cm}$ ) from a high-performance tennis academy volunteered to participate in this study. All participants were nationally ranked (within the top 700 players for their respective age group in the UK). Participants were excluded from the study if they did not have a national ranking and/or were suffering from an injury at the time of testing. Prior to any performance testing, medical screening in the form of a PAR-Q and consent form were completed. Where participants were under 18 years of age, a consent form and information sheet was provided to their parents/ guardians for them to sign on behalf of the individual. Ethical approval was granted by the London Sport Institute, Middlesex University.

\section{Procedures}

All participants were required to complete a total of three trials on each of the testing days for all three CODS assessments, to enable the calculation of both within- and between- session reliability. All trials were completed on an indoor tennis court located at a high-performance tennis academy, with the timing of testing controlled for and thus conducted at the same time of day for each individual respectively. Total time was recorded using electronic timing gates (Brower Timing System, Salt Lake City, Utah, USA), and was recorded to the nearest hundredth of a second. In line with previous CODS research [15], athletes were given a minimum rest period of three minutes between each trial, and three minutes between each test. If/when athletes breached the methodological guidelines for each test (e.g. by failing to reach the line for a COD step), the trial was voided, and an additional trial was conducted following three minutes of rest. Athletes were provided with a standardised warm-up progressive in specificity to the demands of the CODS tests prior to each testing session, inclusive of warm up trials for each of these tests respectively. This was to both minimise the risk of injury, but also to negate any potential warm up effect/ learning effect within trials throughout data collection. The warm-up procedure followed a similar protocol to the RAMP methodology (i.e. raise, activate, mobilise and potentiate), which has been suggested to be an effective protocol to follow prior to physical activity [26].

Spider drill: Considering the spider drill is less well known in the academic literature, a schematic has been provided outlining test protocols (Figure 1). Timing gates were set up at a height of $1 \mathrm{~m}$ for all participants $3 \mathrm{~m}$ behind the baseline, so as to avoid any collisions when returning to the centre point after each sprint. 


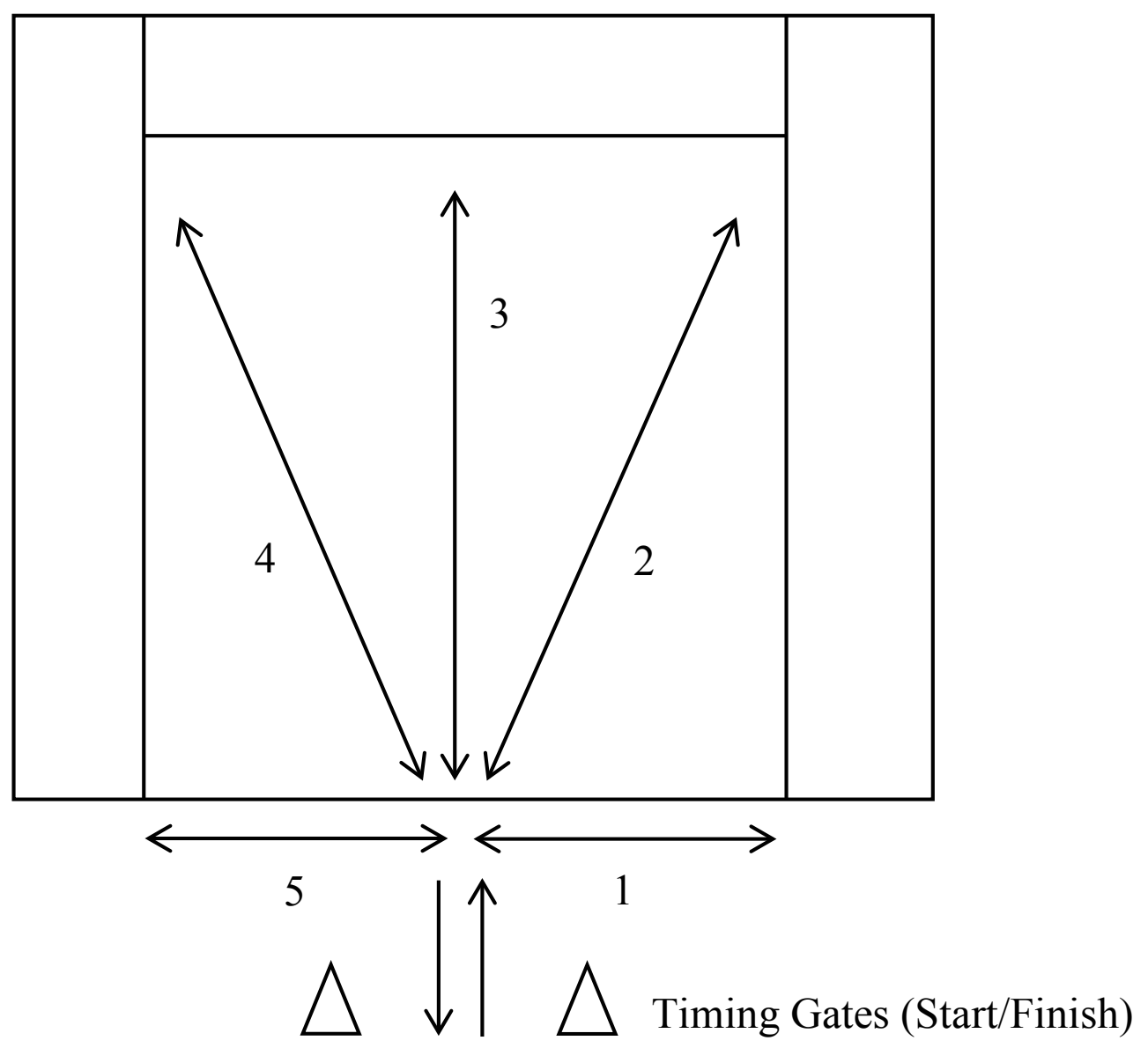

Figure 1: Schematic of the Spider Drill.

On command, participants were instructed to break the beam of the timing gates officially starting the assessment. Participants were required to complete all sprints as outlined in Figure 1, starting with the sprint to the right first (number 1 ) and then working in an anti-clockwise direction thereafter. Sprint numbers 1 and 5 represent a distance of $4.11 \mathrm{~m}$ whilst numbers 2, 3 and 4 each measure $5.49 \mathrm{~m}$. Each sprint required athletes to return to the centre point on the baseline before starting the next. Once the final sprint was completed (returning from 'sprint 5' as viewed in Figure 1), athletes were required to turn right $90^{\circ}$ to complete the three metre sprint through the timing gates completing the test.

Modified T-test: Test protocols were conducted in line with previously validated procedures [23], with timing gates were set at a height of $1 \mathrm{~m}$. Due to the distances typically covered by tennis players (somewhat dictated by court dimensions), the modified version of this test was utilised. Athletes were asked to cover a total distance of $20 \mathrm{~m}$ forming a " $\mathrm{T}$ " shape. A single cone was set up at $5 \mathrm{~m}$ from the timing gates, and then two more cones either side of the first cone at $2.5 \mathrm{~m}$. On command, participants sprinted forward, through the timing gates, and touched the middle cone with their right hand. Participants then side shuffled $2.5 \mathrm{~m}$ to their left (touching the cone with their left hand) and then proceeded to side shuffle $5 \mathrm{~m}$ to the far right cone (this time touching down with their right hand). A side shuffle of $2.5 \mathrm{~m}$ left was then performed back to the centre cone (touching down with their left hand) before back-pedalling $5 \mathrm{~m}$ through the timing gates to complete the test.

Pro-Agility test: Athletes started $0.3 \mathrm{~m}$ just to the left of the timing gates (set at a height of $1 \mathrm{~m}$ ) so as to not risk 'breaking the beam' prior to the commencement of the test. Upon instruction, athletes turned right, cutting the beam and starting the test. This involved a 5-yard sprint to the right whereby all athletes were instructed to touch the cone with their right hand. An immediate $180^{\circ}$ turn was conducted and athletes were required to sprint 10-yards to the opposing cone where they were required to touch down with their left hand. A second $180^{\circ}$ turn was required before sprinting back further 5 -yards, finishing the test as they passed through the timing gates for the third and final time.

\section{Statistical Analysis}

All data analysis was completed using SPSS (V18.0; SPSS, Inc., Chicago, Illinois, USA) and Microsoft Excel ${ }^{\mathrm{TM}}$. Means and Standard Deviations (SD) were calculated from each individuals fastest sprint time for each of the CODS test, for each of the testing days. Normality of the data was established through a Shapiro-Wilk test (due to $<50$ participants), and both within- and betweensession reliability determined via three separate methods: A Coefficient of Variation (CV), a Standard Error of Measurement (SEM), and a two-way random Intraclass 
Table 1: Within-session reliability for each of the three CODS assessments.

\begin{tabular}{|c|c|c|c|c|c|c|c|c|}
\hline \multirow{2}{*}{ CODS Test } & \multicolumn{4}{|l|}{ Session 1} & \multicolumn{4}{|l|}{ Session 2} \\
\hline & Mean \pm SD & CV (\%) & SEM & ICC ( $\pm 95 \%$ CI) & Mean \pm SD & CV (\%) & SEM & ICC ( $\pm 95 \%$ CI) \\
\hline Spider Drill (s) & $16.3 \pm 1.4$ & 2.1 & 0.1 & $0.93(0.74-0.98)$ & $16.2 \pm 1.5$ & 1.8 & 0.07 & $0.95(0.86-0.99)$ \\
\hline Modified T-Test (s) & $6.1 \pm 0.5$ & 3.6 & 0.11 & $0.79(0.52-0.94)$ & $6.2 \pm 0.6$ & 3.6 & 0.1 & $0.83(0.59-0.95)$ \\
\hline Pro-Agility (s) & $5.6 \pm 0.6$ & 3.5 & 0.07 & $0.88(0.66-0.97)$ & $5.5 \pm 0.6$ & 4.1 & 0.15 & $0.69(0.36-0.90)$ \\
\hline
\end{tabular}

Note: Mean values are a representation of the group average from each individuals fastest sprint time.

$\mathrm{s}=$ Seconds; SD = Standard Deviation; CV = Coefficient of Variation; SEM = Standard Error of Measurement; ICC = Intraclass Correlation Coefficient; $\mathrm{Cl}=$ Confidence Interval.

Table 2: Between-session reliability for each of the three CODS assessments.

\begin{tabular}{|c|c|c|c|c|c|c|c|c|}
\hline \multirow{2}{*}{ cODS Test } & \multirow{2}{*}{\begin{tabular}{|l|} 
Session 1 \\
Mean \pm SD \\
\end{tabular}} & \multirow{2}{*}{\begin{tabular}{|l|} 
Session 2 \\
Mean \pm SD
\end{tabular}} & \multirow{2}{*}{\begin{tabular}{|l|} 
Best time \\
Mean \pm SD
\end{tabular}} & \multicolumn{5}{|c|}{ Between sessions } \\
\hline & & & & CV (\%) & SEM (s) & ICC ( $\pm 95 \%$ Cl) & SDD (s) & SDD (\%) \\
\hline Spider & $16.3 \pm 1.4$ & $16.2 \pm 1.5$ & $16.04 \pm 1.42$ & 1.7 & 0.06 & $0.95(0.82-0.99)$ & 0.17 & 1.06 \\
\hline Modified T-Test & $6.1 \pm 0.5$ & $6.2 \pm 0.6$ & $6.07 \pm 0.52$ & 1.2 & 1 & $0.97(0.87-0.99)$ & 0.04 & 0.66 \\
\hline Pro-Agility (s) & $5.6 \pm 0.6$ & $5.5 \pm 0.6$ & $5.39 \pm 0.42$ & 3.7 & 0.13 & $0.66(0.11-0.90)$ & 0.35 & 6.49 \\
\hline
\end{tabular}

Note: Mean values are a representation of the group average from each individuals fastest sprint time.

$\mathrm{s}=$ Seconds; SD = Standard Deviation; CV = Coefficient of Variation; SEM = Standard Error of Measurement; ICC = Intraclass Correlation Coefficient; $\mathrm{Cl}=$ Confidence Interval; SDD = Smallest Detectable Difference.

Correlation Coefficient (ICC) with absolute agreement. This modality of ICC was used due to its capacity to detect absolute agreement within both rank and score. To coincide with previous research, accepted $\mathrm{CV}$ values were set at $<10 \%$ [27], and ICC values reported above 0.75 were accepted as reliable $[23,27,28]$. All measures of within-session reliability were determined through the three trials for each of the CODS tests. All measures of between-session reliability were determined through use of the fastest trial from the pooled trials from each given testing day. SDD was subsequently calculated from the SEM to detect random error scores between testing sessions, and thus to detect meaningful differences. Initially, the SEM was calculated through the equation: $[S D[$ pooled $] * \sqrt{1-\mathrm{ICC}]}$. Subsequently, SDD was calculated through the equation:

$$
[1.96 *(\sqrt{2}) * S E M][29] \text {. }
$$

\section{Results}

The Shapiro-Wilk test concluded all data to be normally distributed ( $p>0.05$ ). Mean, SD, CV, SEM, and ICC values reporting reliability within-sessions are displayed in Table 1 , and between-session reliability reported within Table 2. All CV values (both within- and betweentesting sessions) are below the accepted threshold of $10 \%$ (1.21 - 4.1\%), and SEM values are consistent within tests both within- and between- testing sessions.

The spider drill provided high test retest reliability, as illustrated through the ICC, both within-session (ICC $=0.93$ - 0.95) and between-sessions (ICC $=0.95$ ). The modified t-test also provided high test retest reliability both within- (ICC $=0.79-0.83$ ) and between-sessions (ICC $=0.97$ ). Lower test retest consistency was identified however for the Pro-Agility test, with ICC scores within-session ranging from $0.69-0.88$ (95\% Cl: $0.36-0.97)$, and between-session identified as 0.66 (95\% Cl: 0.11 - 0.9).

All values for SDD are provided within Table 2, with data reported both in absolute terms (seconds), but also as a percentage (\%) of their best time.

\section{Discussion}

To the authors' knowledge, a small sample of literature to date has assessed the reliability and validity of both the modified t-test [23] and pro-agility [15,22] CODS tests, which are theorised to be applicable CODS assessments for youth tennis athletes [11]. However, this is the first study to evaluate the reliability (both within- and between- testing sessions) of the spider drill CODS assessment in comparison to other CODS assessments specifically within elite youth tennis athletes. Overall, our results indicate that the spider drill is a reliable measure of CODS within youth tennis athletes, and can be used with confidence to detect meaningful differences in CODS.

All CODS assessments achieved low typical percentage error, with this both within-session (CV = $1.8-4.1 \%$ ) and between-session (CV $=1.2-3.7 \%$ ). In addition, SEM values were recorded in order to identify which CODS test had the smallest margin of error, as this can assist in detecting true changes when these are greater than the error in the test [15]. Results identified these to be consistent within all tests both within- and between- testing sessions. However, when this information is coupled with test retest consistency data through the ICC, the pro-agility appears to be less reliable than initially anticipated, with both within-session (e.g. testing session 2: ICC $=0.69,95 \% \mathrm{Cl}: 0.36-0.90$ ), and between-sessions (ICC $=0.66,95 \% \mathrm{Cl}: 0.11-0.90)$ reliability falling outside of the proposed threshold. These findings are contradictory to those from Stewart, et al. [15], of whom found high intraday reliability (ICC $=0.9,95 \% \mathrm{Cl}$ : $0.84-0.94$ ) for pro-agility within a sample of physical education students of similar ages to those of the present study (16.7 \pm 0.6 years), and to those of Mayhew, et al. [22], who concluded the pro-agility test to hold strong reliability 
(ICC $=0.8)$. While measures of consistency are important, Stewart, et al. [15] alluded to research which may suggest that within-subject error (i.e. CV and SEM) may be a better measure of reliability, given the importance of detecting error on an individual level to ensure accurate monitoring of performance. The greatest SEM values were also identified however for the pro-agility test both within- and between- sessions (SEM $=0.1-0.11$ $\& 0.13$ respectively), this somewhat surprising given the overall time to complete the test was the shortest of the three.

Previous studies examining the reliability and validity of CODS assessment have employed participants of similar age ranges. Within this study, though all ten participants held a national ranking, the variation in age range can theoretically suggest each participant has had a different level of exposure or experience in terms of playing career; therefore, suggesting some may be more accustomed to the procedures of these assessments than others. Additionally, sample size may have been a limiting factor when comprehending the lower reliability scores within the pro-agility test to those previous suggested within the literature. Whilst this is can only be deemed speculative in nature, it should be noted that all data was identified to be normally distributed, thus allowing for parametric data analysis. As such, the results of this study can be determined applicable to a range of ages in youth tennis athletes, however future studies should look to utilise an athletic population from one age bracket in order to assume the likelihood of similar experience and playing exposure. This will therefore eradicate any issues regarding interage group reliability of such tests. As such, this will allow for a more holistic understanding of both within- and between- age groups, allowing practitioners to more precise when monitoring changes in scores within these CODS tests.

In conclusion, the present study has highlighted that both the spider drill and modified t-test are reliable tests to assess youth tennis athletes CODS ability. In order to be able to generalise these findings outside of youth tennis athletes, a wider population sample would be required. However, the aim of this investigation was solely to provide reliability statistics to support the use of the spider drill as a means of reliably assessing CODS within youth tennis athletes, and the findings support this notion.

With this in mind therefore, the use of the spider drill and modified t-test would be recommended based off their reliability both within- and between- testing sessions, with high test sensitivity to detect change (as illustrated through the SDD). In addition, results from the present study highlight that the pro-agility test may not be the most appropriate CODS test to use for elite youth tennis athletes.

Furthermore, speculation by Roberts, et al. [11] suggests that more than one CODS test should be considered when monitoring changes in CODS, attributing this to the vast complexity of movement patterns completed throughout tennis match play. Given how varied movement patterns occur within the spider drill, when compared to the modified t-test, but also of the notable differences in total time taken to complete these tests, it may be suggested that if practitioners are to use more than one CODS test, that test selection is dictated by the demands of the test, and thus movements that occur. With the spider drill demanding $180^{\circ}$ turns, and the modified t-test incorporating lateral movements, it could be suggested that both of these tests hold strong ecological validity, thus supporting their use within youth tennis athletes. Future research however should look to explore this concept further.

\section{Practical Application}

The results from this study highlight that the spider drill and modified t-test are both reliable measures of CODS within youth tennis athletes, and can be used with confidence to detect meaningful differences in CODS. Strength and conditioning coaches and sport science practitioners could consider changes in excess of $\pm 1.1 \%$ as meaningful when assessing CODS through the aforementioned CODS tests within youth tennis athletes, given that they are familiarised to the demands of such tests before hand.

\section{References}

1. Bangsbo J (1992) Time and motion characteristics of competition soccer. Science of Football 6: 34-42.

2. Deutsch MU, Kearney GA, Rehrer NJ (2007) Time - motion analysis of professional rugby union players during matchplay. J Sports Sci 25: 461-472.

3. Dogramaci SN, Watsford ML, Murphy AJ (2011) Time-motion analysis of international and national level futsal. $J$ Strength Cond Res 25: 646-651.

4. Lockie RG, Schultz AB, Callaghan SJ, Jeffriess MD, Berry SP (2013) Reliability and validity of a new test of change-of-direction speed for field-based sports: the change-of-direction and acceleration test (CODAT). J Sports Sci Med 12: 88-96.

5. SpencerM, Lawrence S, Rechichi C, Bishop D, Dawson B, et al. (2004) Time-motion analysis of elite field hockey with special reference to repeated-sprint activity. Journal of Sports Sciences 22: 843-850.

6. Kovacs MS (2006) Applied physiology of tennis performance. Br J Sports Med 40: 381-385.

7. Kovacs M (2009) Movement for tennis: The importance of lateral training. Strength and Conditioning Journal 31: 77-85.

8. Roetart E, Brown S, Piorkowski P, Woods R (1996) Fitness components among three different levels of elite tennis players. Journal of Strength and Conditioning Research 10: 139-143.

9. Fernandez J, Mendez-Villanueva A, Pluim BM (2006) Intensity of tennis match play. Br J Sports Med 40: 387-391.

10. Fernandez-Fernandez J, Ulbricht A, Ferrauti A (2014) Fitness testing of tennis players: How valuable is it? $\mathrm{Br} \mathrm{J}$ Sports Med 48: 22-31.

11. Roberts T, Turner A, Meyers R (2011) A field based test battery for tennis. Professional Strength and Conditioning Journal 20: 13-22. 
12. Eriksson A, Johansson FR, Bäck M (2015) Reliability and criterion-related validity of the 20 -yard shuttle test in competitive junior tennis players. Open Access $\mathrm{J}$ Sports Med 6: 269-276.

13. Cooke K, Quinn A, Sibte N (2011) Testing speed and agility in elite tennis players. Strength and Conditioning Journal 33 : 69-72.

14. Sheppard JM, Young WB (2006) Agility literature review: Classifications, training and testing. J Sports Sci 24: 919-932.

15. Stewart PF, Turner AN, Miller SC (2014) Reliability, factorial validity, and interrelationships of five commonly used change of direction speed tests. Scand J Med Sci Sports 24: 500-506.

16. Young W, Farrow D (2013) The importance of a sport-specific stimulus for training agility. Strength and Conditioning Journal 35: 39-43.

17. Ben Abdelkrim N, Castagna C, Jabri I, Battikh T, El Fazaa $\mathrm{S}$, et al. (2010) Activity profile and physiological requirements of junior elite basketball players in relation to aerobic-anaerobic fitness. J Strength Cond Res 24: 2330-2342.

18. Bernier M (2003) Perturbation and agility training in the rehabilitation of soccer athletes. Athletic Therapy Today 8: 20-22.

19. Bloomfield J, Ackland T, Elliot B (1994) Applied anatomy and biomechanics in sport. Blackwell Scientific Publications, Melbourne.

20. Lloyd J, Ackland T, Cocham J (1994) Balance and agility. Applied Anatomy and Biomechanics in Sport 211-226.

21. Jeffreys I (2006) Motor learning- applications for agility, part 1. Strength and Conditioning 28: 72-76.
22. Gains GL, Swedenhjelm AN, Mayhew JL, Bird HM, Houser JJ (2010) Comparison of speed and agility performance of college football players on field turf and natural grass. $J$ Strength Cond Res 24: 2613-2617.

23. Sassi RH, Dardouri W, Yahmed MH, Gmada N, Mahfoudhi ME, et al. (2009) Relative and absolute reliability of a modified agility T-test and its relationship with vertical jump and straight sprint. J Strength Cond Res 23: 1644-1651.

24. Chandler TJ (1995) Exercise training for tennis. Clin Sports Med 14: 33-46.

25. Kovacs M, Pritchett R, Wickwire P, Green J, Bishop P (2007) Physical performance changes after unsupervised training during the autumn/spring semester break in competitive tennis players. Br J Sports Med 41: 705-710.

26. Jeffreys I (2006) Warm-up revisited - the "ramp" method of optimising performance preparation. Professional Strength and Conditioning Journal 11: 15-19.

27. Cormack S, Newton R, McGuigan M, Doyle T (2008) Reliability of measures obtained during single and repeated countermovement jumps. Int J Sports Physiol Perform 3: 131-144.

28. Weir JP (2005) Quantifying test-retest reliability using the intraclass correlation coefficient and the SEM. J Strength Cond Res 19: 231-240.

29. Dos Santos T, Thomas C, Comfort P, McMahon J, Jones $P$, et al. (2017) Between-session reliability of isometric midthigh pull kinetics and maximal power clean performance in male youth soccer players. J Strength Cond Res. 